\title{
Possible inhibitors of ACE2, the receptor of 2019-nCoV
}

Chuanbo Huang ${ }^{1}$, Xiangwen $\mathrm{Ji}^{1}$, Wanlu Zhou ${ }^{2}$, Fenghong Zhang ${ }^{2}$, Liang Wang ${ }^{2}$, Qinghua $\mathrm{Cui}^{1 *}$

${ }^{1}$ Department of Biomedical Informatics, Department of Physiology and Pathophysiology, Center for Noncoding RNA Medicine, MOE Key Lab of Cardiovascular Sciences, School of Basic Medical Sciences, Peking University, 38 Xueyuan Rd, Beijing, 100191, China ${ }^{2}$ Co., Ltd of JeaMoon Technology, 6 Rd Middle Zuojiazhuang, Beijing, 100028, China.

*To whom the correspondence should be addressed:

Dr. Qinghua Cui

Tel: 8610-82801001

Email: cuiqinghua@hsc.pku.edu.cn

Keywords: 2019-nCoV, therapeutic strategies, drug, ACE2 


\section{Abstract}

Most recently, an outbreak of severe pneumonia caused by the infection of 2019-nCoV, a novel coronavirus first identified in Wuhan, China, imposes serious threats to public health. Many important aspects about 2019-nCoV remain largely unknown, among which, the limitation of antiviral therapies represents one of the most critical problems. More recently, it was confirmed that human ACE2 is the receptor for the entry of 2019-nCoV into lower respiratory tract epithelial cells. Give this observation, it is thus expected that the virus could be inhibited if we decrease the expression of ACE2. Here by screening two databases, Connectivity Map (CMap) and our JeaMoon Map (JMap), we identified a number of candidate agents that decrease ACE2 expression. CMap analysis identified 5 compounds, among which, Azathioprine is a possible therapeutic strategy for anti-2019-nCoV. Moreover, JMap analysis revealed a number of comounds, biologics, and traditional Chinese medicine, among which, Andrographis, Urtica, Sambucus, Astragalus, valproic acid, butyrate, and epoxomicin represent the most significant and possible strategies for anti-2019-nCoV therapies. This study provides a number of clues and possible therapeutic strategies for 2019-nCoV prevention and treatment.

\section{Introduction}

In the end of 2019 , some patients with severe febrile respiratory illness were admitted to a hospital in Wuhan, Hubei Province, China. However, no known pathogens were detected in the clinical specimens from these patients[1]. Quickly, sequencing to the RNA extracted from bronchoalveolar-lavage fluid from the patients revealed a novel coronavirus, designated as 
2019-nCoV, which belong to the lineage B of the genus betacoronavirus[1]. The virus seems spreads rapidly. As of January 24, 2020, more than 800 cases have been reported and the mortality rate is about 3\%[2]. China made a quick response including active case finding, improve public awareness, and sharing sequence information, which provides helps in the patient monitoring, diagnostics and prevention of 2019-nCoV[3,4]. However, a number of key questions remain to be explored[4], for example the virus's origin and cross-species transmission, its ability of spread, the extend of interhuman transmission, and the spectrum of clinical disease.

To address these important questions and issues, the global scientific community also has a quick response. For example, the detection of 2019-nCoV has achieved good advances[5]. In addition, clinical features of patients infected with 2019-nCoV were revealed[6]. Moreover, a number of computational analysis and modeling have been performed to explore the origin, infectivity, and cross-species transmission[7,8,9], evolution[10], transmission patterns and rules[11,12,13,14,15]. These studies provided helps in better understanding the origin, evolution, and transmission of 2019-nCoV.

However, one of the most important questions is that current treatments for 2019-nCoV are empirical[6] and there are still no efficient antiviral therapies[2]. Moreover, we also failed to find publications for the development of therapeutic strategies for 2019-nCoV. Recently, a team led by Profs. Zihe Rao and Haitao Yang at ShanghaiTech University reported the structure of 2019-nCoV proteinase (M ${ }^{\text {pro }}$, also called 3CL ${ }^{\text {pro }}$, PDB ID: 6LU7). Based on the structure, $\sim 20$ research teams from Shanghai Institute of Materia Medica (SIMM), Chinese Academy of Sciences (CAS) and ShanghaiTech University together quickly identified 30 
potential chemical compounds and natural products with anti-2019-nCoV activity using virtual screening and enzymology, as stated in a News at the SIMM web site on January 25, 2020 (http://www.simm.ac.cn/xwzx/kydt/202001/t20200125_5494417.html, in Chinese). However, before the identification of efficient drugs, the development of more possible therapeutic strategies is still emergently needed. Like SARS-CoV, 2019-nCoV also belongs to the lineage B of the genus betacoronavirus. It thus appears that as SARS-CoV, the human angiotensin-converting enzyme 2 (ACE2) is also the receptor for 2019-nCoV's entry into lower respiratory tract epithelial cells[2]. This was recently confirmed by Letko and Munster[16]. Given that ACE2 is a key human gene for 2019-nCoV' infection to human, it is thus expected that approaches decreasing ACE2 expression could has anti-2019-nCoV activities.

Based on the above observations, here we screened potential agents decreasing ACE2 expression using two databases, Connectivity Map (CMap) developed by Broad Institute and JeaMoon Map (JMap) developed by Co., Ltd of JeaMoon Technology. As a result, CMap analysis identified 5 compounds and JMap analysis identified a number of agents including compounds, traditional Chinese medicine (TCM), and biologics that can functionally decrease the expression of ACE2, suggesting that these agents could be possible possible therapeutic strategies for the prevention and treatment of 2019-nCoV infection.

\section{Methods}

\section{Datasets of gene expression profiles induced by drugs and other agents}

From Connectivity Map[17] (CMap, version 2.0), we obtained the gene expression profiles 
induced by $\sim 1000$ compounds. We obtained nearly 2000 gene expression profiles induced by a variety of agents including compounds, traditional Chinese medicine, environmental factors, exercise etc from JeaMoon Map (JMap), which is a commercial database developed by Co., Ltd of JeaMoon Technology.

\section{Screening agents decreasing ACE2 expression}

We used the normalized gene expression datasets. A cutoff of fold change (FC) 2.0 was selected to determine whether ACE2 is significantly decreased by a putative agent or not. Then, we screened CMap and JMap using a single-gene screening module in the JeaMoon DrugMine software platform.

\section{Results}

\section{The CMap compounds that decrease ACE2 expression}

As a result, CMap analysis identified 5 compounds that functionally decrease the expression level of ACE2 (Fig 1), including Fulvestrant (FC=3.16), Pirinixic Acid (FC=3.13), AG-013608 ( $\mathrm{FC}=2.72)$, Azathioprine $(\mathrm{FC}=2.08)$, and Staurosporine $(\mathrm{FC}=2.01)$, suggesting that these compound could relatively block the entry of 2019-nCoV into lower respiratory tract epithelial cells. After a literature search, it seems that only Azathioprine is possibly used for anti-2019-nCoV. Azathioprine is used for the prevention of organ rejection and rheumatoid arthritis by weakening the immune system as an immunosuppressant. The severe pneumonia could be an outcome of extreme immune response of our body to 2019-nCoV. The information for AG-013608 is quite limited and we have no idea about its possibility to be an anti-2019-nCoV therapy. The possibility of the other three compounds is low because of 
potential health hazard, toxicity, and side effects.

\section{The JMap agents that decrease ACE2 expression}

As a result, JMap analysis identified a number of agents that functionally decrease the expression level of ACE2 (Fig 2). These agents include traditional Chinese Medicine (TCM), compound, and biologics (Fig 2). The 4 TCM showed significant ability to decrease ACE2 expression, especially Andrographis $(\mathrm{FC}=21.57)$, Urtica $(\mathrm{FC}=11.59)$, and Sambucus (5.99). Indeed, some studies reported potential effects of anti-pneumonia for active ingredients from Andrographis[18,19], Urtica[20], Sambucus[21], and Astragalus[22]. This finding suggests that the four TCM could be possible anti-2019-nCoV therapies. For the small molecules, we found literature that some predicted compounds can mitigate pneumonia or lung injury, including valproic acid[23], butyrate[24], epoxomicin[25], and emetine[26]. Because arsenic trioxide shows significant toxicity, it should be carefully to test its possibility. Interestingly, nicotine shows an ability of decreasing ACE2 expression, suggesting a possible role of anti-2019-nCoV. This finding may be consistent with our previous report that smoking-inhibited miRNAs are significantly associated with SARS[27]. For the biologics, previous reports supported the prediction for lipopolysaccharide[28]. We previously found oxLDL has protective roles in influenza virus infection[29], suggesting that oxLDL could be a protective factor for 2019-nCoV infection, as predicted here.

\section{DISCUSSION}

The recent outbreak 2019-nCoV infection brings a new severe problem for public health. The unavailability of efficient therapeutic strategies is one of the most important questions at 
present. To address this issue, here we identified a number of possible therapeutic strategies by screening CMap and JMap for potential agents decreasing ACE2 expression based on the observation that ACE2 is one key gene for the entry of 2019-nCoV into human cells. This study provides clues for possible therapeutic strategies to fight 2019-nCoV. However, when trying these therapeutic strategies in cases with 2019-nCoV infection, some issues should be considered. Firstly, it is difficult to predict a suitable dosage for the identified agents. Therefore, the dosage should be explored in practice in human cases. Moreover, people in different stages of infection may be sensitive and safe to different dosages. Secondly, the modes of drug delivery should be explored too. Different drugs may take different modes of drug administration for anti-2019-nCoV. In addition, the diseased location is in the respiratory tract, atomization aspirate could get better outcome. Thirdly, combinations of two or more of the identified agents could achieve better therapeutic outcome. Finally, the present study is to provide clues for 2019-nCoV therapy. Practice and operations based on the results of this study should be performed according to specific conditions.

\section{References}

[1] N. Zhu, D. Zhang, W. Wang, X. Li, B. Yang, J. Song, X. Zhao, B. Huang, W. Shi, R. Lu, P. Niu, F. Zhan, X. Ma, D. Wang, W. Xu, G. Wu, G.F. Gao, W. Tan, I. China Novel Coronavirus, T. Research, A Novel Coronavirus from Patients with Pneumonia in China, 2019, N Engl J Med (2020).

[2] S. Perlman, Another Decade, Another Coronavirus, N Engl J Med (2020).

[3] D.S. Hui, I.A. E, T.A. Madani, F. Ntoumi, R. Kock, O. Dar, G. Ippolito, T.D. McHugh, Z.A. Memish, C. Drosten, A. Zumla, E. Petersen, The continuing 2019-nCoV epidemic threat of novel coronaviruses to global health - The latest 2019 novel coronavirus outbreak in Wuhan, China, Int J Infect Dis 91 (2020) 264-266.

[4] V.J. Munster, M. Koopmans, N. van Doremalen, D. van Riel, E. de Wit, A Novel Coronavirus Emerging in China - Key Questions for Impact Assessment, N Engl J Med (2020).

[5] N. Zhang, L. Wang, X. Deng, R. Liang, M. Su, C. He, L. Hu, Y. Su, J. Ren, F. Yu, L. Du, S. Jiang, Recent advances in the detection of respiratory virus infection in humans, J Med Virol 
(2020).

[6] C. Huang, Y. Wang, X. Li, L. Ren, J. Zhao, Y. Yu, L. Zhang, G. Fan, J. Xu, X. Gu, Z. Cheng, T. Yu, J. Xia, Y. Wei, W. Wu, X. Xie, W. Yin, H. Li, M. Liu, Y. Xiao, H. Gao, L. Guo, J. Xie, G. Wang, R. Jiang, Z. Gao, Q. Jin, J. Wang, B. Cao, Clinical features of patients infected with 2019 novel coronavirus in Wuhan, China, The Lancet 0 (2020) 10.

[7] N. Dong, X. Yang, L. Ye, K. Chen, E.W.-C. Chan, M. Yang, S. Chen, Genomic and protein structure modelling analysis depicts the origin and infectivity of 2019-nCoV, a new coronavirus which caused a pneumonia outbreak in Wuhan, China, bioRxiv (2020) 2020.2001.2020.913368.

[8] Q. Guo, M. Li, C. Wang, P. Wang, Z. Fang, J. tan, S. Wu, Y. Xiao, H. Zhu, Host and infectivity prediction of Wuhan 2019 novel coronavirus using deep learning algorithm, bioRxiv (2020) 2020.2001.2021.914044

[9] W. Ji, W. Wang, X. Zhao, J. Zai, X. Li, Homologous recombination within the spike glycoprotein of the newly identified coronavirus may boost cross-species transmission from snake to human, J Med Virol (2020).

[10] D. Benvenuto, M. Giovannetti, A. Ciccozzi, S. Spoto, S. Angeletti, M. Ciccozzi, The 2019-new coronavirus epidemic: evidence for virus evolution, bioRxiv (2020) 2020.2001.2024.915157.

[11] T. Chen, J. Rui, Q. Wang, Z. Zhao, J.-A. Cui, L. Yin, A mathematical model for simulating the transmission of Wuhan novel Coronavirus, bioRxiv (2020) 2020.2001.2019.911669.

[12] Z. Chen, W. Zhang, Y. Lu, C. Guo, Z. Guo, C. Liao, X. Zhang, Y. Zhang, X. Han, Q. Li, J. Lu, From SARS-CoV to Wuhan 2019-nCoV Outbreak: Similarity of Early Epidemic and Prediction of Future Trends, bioRxiv (2020) 2020.2001.2024.919241.

[13] J.M. Read, J.R. Bridgen, D.A. Cummings, A. Ho, C.P. Jewell, Novel coronavirus 2019-nCoV: early estimation of epidemiological parameters and epidemic predictions, medRxiv (2020) 2020.2001.2023.20018549.

[14] J. Riou, C.L. Althaus, Pattern of early human-to-human transmission of Wuhan 2019-nCoV, bioRxiv (2020) 2020.2001.2023.917351

[15] S. Zhao, J. Ran, S.S. Musa, G. Yang, Y. Lou, D. Gao, L. Yang, D. He, Preliminary estimation of the basic reproduction number of novel coronavirus (2019-nCoV) in China, from 2019 to 2020: A data-driven analysis in the early phase of the outbreak, bioRxiv (2020) 2020.2001.2023.916395.

[16] M. Letko, V. Munster, Functional assessment of cell entry and receptor usage for lineage B $\beta$-coronaviruses, including 2019-nCoV, bioRxiv (2020) 2020.2001.2022.915660.

[17] J. Lamb, E.D. Crawford, D. Peck, J.W. Modell, I.C. Blat, M.J. Wrobel, J. Lerner, J.P. Brunet, A. Subramanian, K.N. Ross, M. Reich, H. Hieronymus, G. Wei, S.A. Armstrong, S.J. Haggarty, P.A. Clemons, R. Wei, S.A. Carr, E.S. Lander, T.R. Golub, The Connectivity Map: using gene-expression signatures to connect small molecules, genes, and disease, Science 313 (2006) 1929-1935.

[18] G. Zhang, C. Jiang, N. Xie, Y. Xu, L. Liu, N. Liu, Treatment with andrographolide sulfonate provides additional benefits to imipenem in a mouse model of Klebsiella pneumoniae pneumonia, Biomed Pharmacother 117 (2019) 109065.

[19] T. Zhang, L. Zhu, M. Li, Y. Hu, E. Zhang, Q. Jiang, G. Han, Y. Jin, Inhalable Andrographolide-beta-cyclodextrin Inclusion Complexes for Treatment of 
Staphylococcus aureus Pneumonia by Regulating Immune Responses, Mol Pharm 14 (2017) 1718-1725.

[20] S. Arefani, S.M.M. Mehran, H. Moladoust, M.R. Norasfard, A. Ghorbani, M. Abedinzade, Effects of standardized extracts of Lamium album and Urtica dioica on rat tracheal smooth muscle contraction, J Pharmacopuncture 21 (2018) 70-75.

[21] J.R. Weng, C.S. Lin, H.C. Lai, Y.P. Lin, C.Y. Wang, Y.C. Tsai, K.C. Wu, S.H. Huang, C.W. Lin, Antiviral activity of Sambucus FormosanaNakai ethanol extract and related phenolic acid constituents against human coronavirus NL63, Virus Res 273 (2019) 197767.

[22] Z.C. Yang, Z.H. Qu, M.J. Yi, C. Wang, N. Ran, N. Xie, P. Fu, X.Y. Feng, Z.D. Lv, L. Xu, Astragalus extract attenuates allergic airway inflammation and inhibits nuclear factor kappaB expression in asthmatic mice, Am J Med Sci 346 (2013) 390-395.

[23] G. Kasotakis, M. Galvan, E. King, B. Sarkar, A. Stucchi, J.P. Mizgerd, P.A. Burke, D. Remick, Valproic acid mitigates the inflammatory response and prevents acute respiratory distress syndrome in a murine model of Escherichia coli pneumonia at the expense of bacterial clearance, J Trauma Acute Care Surg 82 (2017) 758-765.

[24] J. Liu, G. Chang, J. Huang, Y. Wang, N. Ma, A.C. Roy, X. Shen, Sodium Butyrate Inhibits the Inflammation of Lipopolysaccharide-Induced Acute Lung Injury in Mice by Regulating the Toll-Like Receptor 4/Nuclear Factor kappaB Signaling Pathway, J Agric Food Chem 67 (2019) 1674-1682.

[25] J.M. Albright, J. Romero, V. Saini, S.U. Sixt, M.D. Bird, E.J. Kovacs, R.L. Gamelli, J. Peters, M. Majetschak, Proteasomes in human bronchoalveolar lavage fluid after burn and inhalation injury, J Burn Care Res 30 (2009) 948-956.

[26] C.R. Wilson, Broncho-Pneumonia in Children Treated by Injections of Emetine Hydrochloride, Br Med J 1 (1928) 844-845.

[27] B. Shi, H. Gao, T. Zhang, Q. Cui, Analysis of plasma microRNA expression profiles revealed different cancer susceptibility in healthy young adult smokers and middle-aged smokers, Oncotarget 7 (2016) 21676-21685.

[28] J. Zhao, C. Wohlford-Lenane, J. Zhao, E. Fleming, T.E. Lane, P.B. McCray, Jr., S. Perlman, Intranasal treatment with poly $(1 * \mathrm{C})$ protects aged mice from lethal respiratory virus infections, J Virol 86 (2012) 11416-11424.

[29] C. Huang, W. Yang, J. Wang, Y. Zhou, B. Geng, G. Kararigas, J. Yang, Q. Cui, The DrugPattern tool for drug set enrichment analysis and its prediction for beneficial effects of oxLDL on type 2 diabetes, J Genet Genomics 45 (2018) 389-397. 


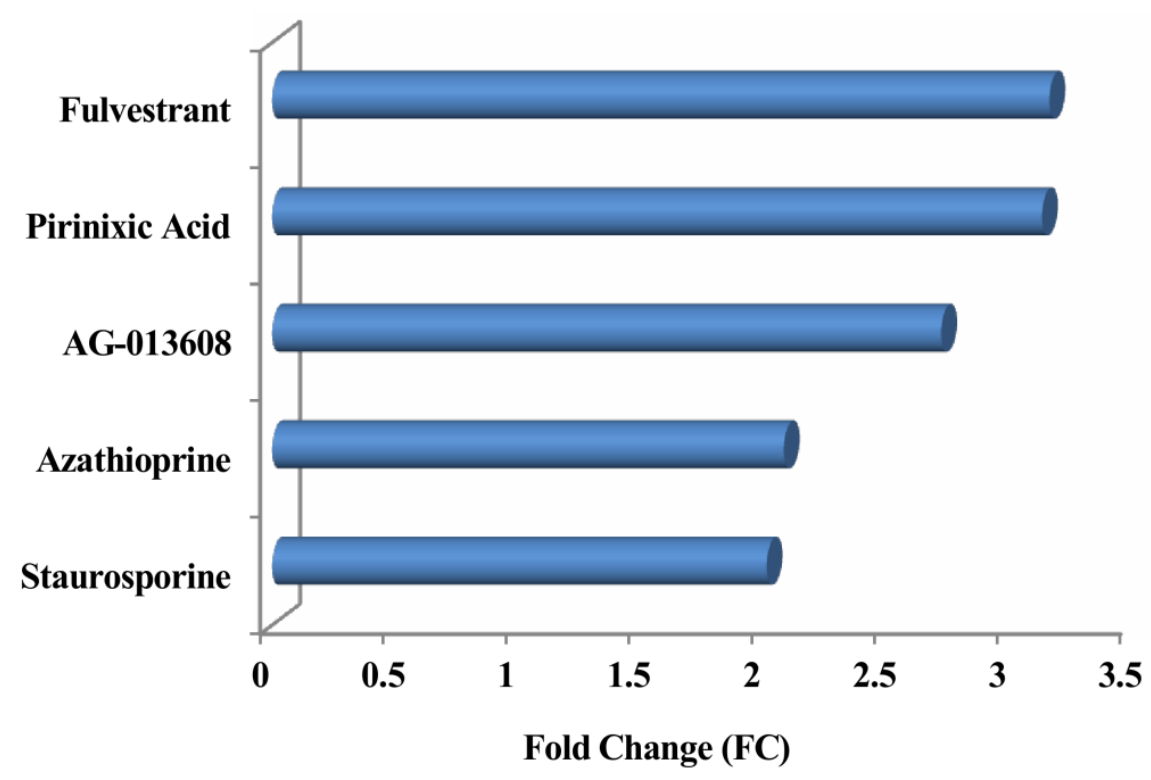

Figure 1. CMap analysis identified five compounds that decrease ACE2 expression. The length of each bar represents the fold change (FC) of ACE2 induced by the corresponding compound. Because ACE2 is one key gene for the entry of 2019-nCoV into human cell, compounds that can decrease ACE2 expression are thus considered to be possible therapeutic strategies for anti-2019-nCoV. 


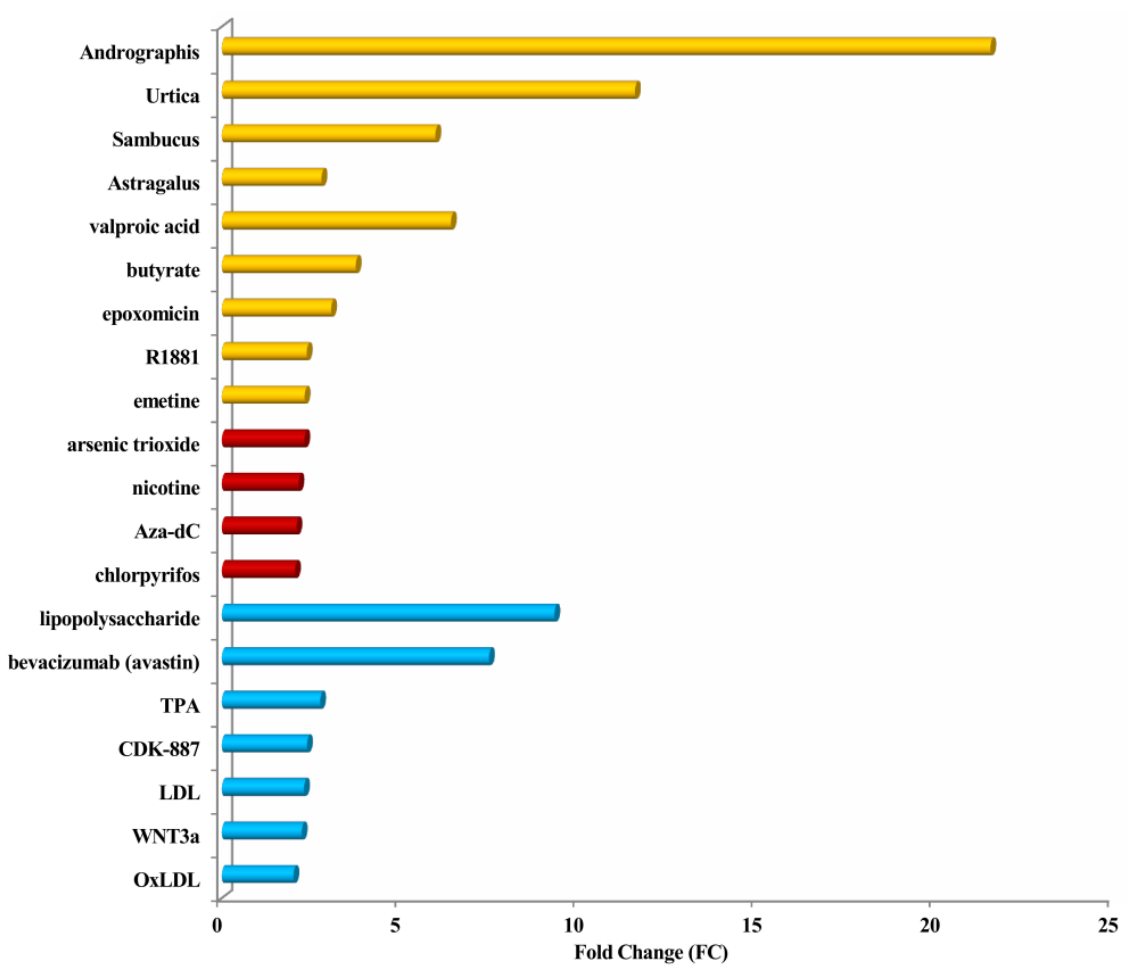

Figure 2. JMap analysis identified 36 agents that decrease ACE2 expression. The length of each bar represents the fold change (FC) of ACE2 induced by the corresponding agent. Because ACE2 is one key gene for the entry of 2019-nCoV into human cell, agents that can decrease ACE2 expression are thus considered to be possible therapeutic strategies for anti-2019-nCoV. The agents include traditional Chinese medicine (TCM), Compound, and Biologics, which are shown in different colors. 